\title{
Comparative analysis of intrauterine device placement or estrogen therapy to patients after hysteroscopic uterine septum resection.
}

\author{
Jing Xue, Hui Zhang, Min Cui, Na Li, Ming Liu, Yulan Mu* \\ Department of Obstetrics and Gynecology, Shandong Provincial Hospital Affiliated to Shandong University, Jinan, \\ Shandong Province, PR China
}

\begin{abstract}
Whether the implement of Intrauterine Device (IUD) and/or Estrogen Replacement Therapy (ERT) is required for preventing intrauterine adhesion after transcervical hysteroscopic resection of uterine septum (TCRS) remains unclear, here, we report a retrospective cohort analysis of the outcomes of patients who had received different treatments after TCRS. Among the 223 cases received TCRS and/or postoperative treatments, the group A, B and C received TCRS at periods 2002-2007, 2007-2009 and 2009-2011 respectively. The group A of 74 patients was placed IUD for $2 \sim 3$ months after operation. Group B of 63 patients was given ERT treatment for $2 \sim 3$ cycles. Group $C$ of 86 patients received neither IUD nor ERT. All of the patients were checked hysteroscopically for uterine cavity adhesions during the period of the third to the seventh days after the second menstruation. All pregnancies occurring during the observation period were recorded. The TCRS succeeded for all patients. None of them needed a twostage operation. Cavity membrane adhesion of uterine cavity formed in 3 cases, 2 cases and 2 cases in the groups A, B, C respectively. 194 of 223 patients got pregnant within one year after TCRS. 160 children had been delivered successfully. The live birth rate was $71.7 \%(160 / 223)$. The abortion rates after operation were 10 cases, 7 cases and 9 cases in A, B, C group respectively. Statistical analysis showed no significant differences in uterine adhesion, pregnancy rate, abortion frequency, live birth rate and the rate of cesarean section among the three groups. However, the postoperational cost of group $\mathrm{C}$ was significantly lower than that of groups A and B. In short, neither postoperational IUD placement, nor estrogen therapy, nor the combination of IUD and ERT demonstrated any discernible clinical benefits to the patients in preventing intrauterine adhesions and facilitating pregnancy after TCRS.
\end{abstract}

Keywords: Uterine septum, Intrauterine adhesion, Transcervical hysteroscopic resection, Intrauterine device, Estrogen therapy.

Accepted on April 03, 2018

\section{Introduction}

Uterine malformations are the most common defects of the female reproductive system, occurring with the incidence of about $4 \%$ in the general population of women [1]. It is reported that uterine septum, an important type of uterus defects, occurred with the incidence of $24 \%$ and constituted a risk of infertility, miscarriage and complications of pregnancy as well as childbirth [2,3]. Abortion rate in patients with uterine septum is as high as $77.4 \%$ with only $6-28 \%$ of the live birth rate $[4,5]$. Therefore, more than $50 \%$ patients need uterine septum resection before pregnancy. The incidence of septate uterus, type $\mathrm{V}$ of female genital tract malformation, is about $0.73-12 \%$ and accounts up to $80-90 \%$ of uterine malformation, caused by the absorption disorder after the fusion of bilateral accessory of embryonic nephridium [6,7]. Being less invasive, the hysteroscopy uterine septum resection (TCRS) is currently considered as an ideal and standard operation to resect uterine septum. Pregnancy can be expected as early as 2-3 months after operation, and the baby can be delivered vaginally [8].

Intrauterine adhesion, one of the hysteroscopic complications, often occurs after hysteroscopic adhesion resection (TCRA), endometrial resection (TCRE) and electric multiple submucous leiomyoma resection (TCRM). Only $2-5.3 \%$ of patients received TCRS suffer from intrauterine adhesion [9]. In order to prevent the occurrence of intrauterine adhesions, postoperational placement of IUD and/or estrogen therapy is widely used in patients received TCRS. However, the benefit of IUD and/or estrogen therapy in preventing uterine adhesion and facilitating pregnancy is not yet fully evaluated.

In the current study, our retrospective cohort analysis of the outcomes of 264 patients who had received different treatments after TCRS showed that IUD placement and/or estrogen treatment don't show any discernible clinical benefits in preventing intrauterine adhesions and facilitating pregnancy post TCRS. 


\section{Materials and Methods}

This study has been approved by the Institutional Review Board of Shandong Provincial Hospital. Informed consent was obtained from all participants prior to operation. Each author will agree to submit a statement from our Ethics Committee or Institutional Review Board indicating the approval of the research. The written informed consent was given by participants for the clinical records to be used in this study.

\section{Project design}

The retrospective cohort study covers the patients admitted to Shandong Provincial Hospital affiliated to Shandong University during 2002 to 2012. When TCRS was first introduced to treat uterine septum, IUD was used to prevent uterine adhesion. 4 years later, IUD placement was replaced by estrogen therapy as a postoperational approach to prevent uterine adhesion. Informed consent was obtained from all participants prior to operation, which was approved by the institutional review board of Shandong Provincial Hospital Affiliated to Shandong University.

All enrolled patients with uterine septum were acknowledged by detailed information about the TCRS and IUD/estrogen therapy and gave written consent to choose postoperational approaches. All patients with uterine septum also received routine preoperative examinations, including gynaecological endocrine, thyroid function, anti-cardiolipin antibodies, and antinuclear antibody; meanwhile those with recurrent spontaneous abortion were also examined for chromosomerelated disorders [10,11]. From 2002 to 2012, 264 patients received TCRS in Shandong Provincial Hospital affiliated to Shandong University were enrolled in this study and 262 patients had completely follow-up information. Patients aging from 23-37 y old with an average age of $27.8 \mathrm{y}$ old. And 223 of 262 patients had spontaneous abortion history, in which 198 cases had spontaneous abortion for 2-7 times with gestational age of 10 to 20 weeks. Thirty-seven patients have complete septate uterus and 157 patients have incomplete uterine septum. Twenty-nine patients have both complete septate uterus and vagina mediastinum.

The operations were performed within 3-10 d after menstruation under intravenous anaesthesia and B ultrasound monitoring. The operation of early cases and complicated cases were conducted under laparoscopic exploration. Patients received TCRS were divided into 3 groups according to the postoperative therapy for preventing intrauterine adhesions formation. The group A of 74 patients received TCRS and placement of IUD for 2-3 months after operation. Group B of 63 patients was given estrogen therapy (Climen, 1 tablet/d, Bayer Healthcare Company Limited, Guangzhou, China) for 2-3 cycles post operation. Climen was given according to the menstrual cycle, that is, a 28 -d sequential therapy combing estradiol valerate $2 \mathrm{mg} / \mathrm{d}$ (white tablets) from day 1 to day 11 , and estradiol valerate $2 \mathrm{mg} / \mathrm{d}$ plus cyroterone acetate $1 \mathrm{mg} / \mathrm{d}$ (pink tablets) from day 12 to day 21, followed by a 7-d treatment-free interval. Group $\mathrm{C}$ of 86 patients received only
TCRS neither IUD nor estrogen therapy was given after operation.

\section{Equipment setup}

OLYMPUS bipolar plasma kinetic resection: Electric coagulation power $280 \mathrm{~W}$, power $80 \mathrm{~W}$; the distending liquid applied is $0.9 \% \mathrm{NaCl}$ and the distending pressure is 100 $\mathrm{mmHg}$. Firstly, remove mediastinal tissue from the turn to the end of palace with diathermy loop electrical excision, then put the needle electrode into smooth mediastinal tissue at the end of the palace to obtain neat, symmetrical shape, and the bilateral tubal openings lying at the same level. During operation, B ultrasound monitoring was used to make sure the distance between the bottom of mediastinal margin and the fundal serosal layer was $1 \mathrm{~cm}$, and to make sure the distance between mediastinal tangent and the front wall serosa layer as close as possible. Oxytocin was used when necessary if more intraoperative bleeding occurred.

\section{Perioperational treatment and management}

The group A of 74 patients received TCRS and placement of IUD for 2-3 months after operation. Group B of 63 patients was given Estrogen therapy (Climen) treatment for 2-3 cycles post operation. Group C of 86 patients received TSCR alone, neither IUD nor Estrogen therapy was given after operation. For patients receive complete mediastinum resect and with wider $(>2.0 \mathrm{~cm})$ cutting edge, 12 Foley catheter with $4-6 \mathrm{~mL}$ of saline was placed temporarily, which was removed $48 \mathrm{~h}$ after operation.

All patients received antibiotics $30 \mathrm{~min}$ before and $24 \mathrm{~h}$ after operation or $48 \mathrm{~h}$ if necessary. To soften cervix, $400 \mu \mathrm{g}$ misoprostol was placed in posterior fornix of vagina $12 \mathrm{~h}$ before operation and $200 \mu \mathrm{g}$ misoprostol was taken orally $2 \mathrm{~h}$ before operation. Medicine for promoting uterine contraction and hemostatic drugs may be used post operation. Climen was given for 1 cycle. For patients received complete uterine septate resect or with mediastinal margin bottom opening wider than $2.0 \mathrm{~cm}$, or combined with vagina mediastinum resect, Climen was given 3 cycles. Patients with polycystic ovary syndrome, hyperprolactinemia, thyroid disease, inadequate luteal function, cervix relaxation, anticardiolipin antibody syndrome were treated accordingly. Patients with unknown reasons of recurrent spontaneous abortion were given Philharmonic dimension (1 tablet/d), dydrogesterone $(20 \mathrm{mg} / \mathrm{d})$ and aspirin $(25 \mathrm{mg} / \mathrm{d})$ for 1 month before pregnancy and 3 months after pregnancy.

\section{Patients follow-up}

Follow-up was obtained by telephone, mail and electronic-mail from the first month post-operation to the first pregnancy or the delivery of the first child after surgery. The follow-up time is 15-21 months with an average of $14 \pm 3$ months. Follow-up information includes menstrual quality, dysmenorrhea, menstrual cycle, pregnancy, abortion, premature delivery, childbirth complication, delivery path, weight and Apgar score 
of child. The first outpatient examination was performed on the third day of the menstrual cycle. The second outpatient examination was performed on the third day after the second menstruation using ultrasonography and hysteroscopy. 2 or 3 months after TCRS, removal of IUDs depends on the recovery of endometrium examined by hysteroscopy. Patients were recommended to be pregnant at least 2-3 months after surgery.

\section{Statistical analysis}

Statistical analysis was performed using SPSS 17 . Values are expressed as mean \pm SD. Differences between two and multiple groups were determined by analysis of student t-test and $\mathrm{X}^{2}$ test. A p-value $<0.05$ was considered statistically significant.

\section{Results}

\section{Comparison of the operation time, the amounts of bleeding and perfusion during operation in three groups of patients}

The general information, including operation time, the amounts of perfusion and bleeding during operation, are shown in Table 1. There is no significant difference in operation times between group A and group B, however, the operation time of group $\mathrm{C}$ is significantly shorter than that in group A and B $(p<0.05)$. As shown in Table 1, the amounts of perfusion and bleeding in group $\mathrm{C}$ are obviously less than that in group $\mathrm{A}$ and $\mathrm{B}$ $(p<0.05)$. However, no significant difference exists between group $A$ and $B(p>0.05)$.

Table1. Parameters of TCRS.

\begin{tabular}{llllll}
\hline Groups & Cases & Age & Duration $(\mathrm{min})$ & Perfusion $(\mathrm{ml})$ & Bleeding $(\mathrm{ml})$ \\
\hline A & 74 & 27.7 & $32 \pm 6.4$ & $1310 \pm 147.7$ & $30 \pm 4.8$ \\
\hline B & 63 & 27.2 & $25 \pm 4.3$ & $920 \pm 128.5$ & $27 \pm 4.4$ \\
\hline C & 86 & 26.8 & $17 \pm 3.1^{\text {a }}$ & $730 \pm 117.9^{*}$ & $13 \pm 2.6^{*}$ \\
\hline
\end{tabular}

* $p<0.05$ vs. group A.

\section{Comparison of the effects of postoperational therapies on intrauterine adhesion formation, pregnancy, abortion and live birth}

264 patients received TCRS are followed by different postoperational therapies, in which 41 cases are excluded for incomplete clinical information. In hysteroscopy examination, intrauterine cavity adhesion formation is developed in $3(4.1 \%$, $3 / 74), 2(3.2 \%, 2 / 63)$ and $2(2.3 \%, 2 / 86)$ cases in group A, B and $\mathrm{C}$, respectively, which is consistent with the results reported by Tonguc et al. (Table 2) [12]. In group B, introduction of IUD results in minor vaginal bleeding and abdominal discomfort. Patients in group $\mathrm{C}$ receiving neither IUD nor estrogen therapy do not show severe complications.

Table 2. Comparison of adhesion rate with Tonguc et al.'s study [12].

\begin{tabular}{lll}
\hline Treatments & This study & Tonguc et al.'s study \\
\hline IUD & $3 / 74(4.1 \%)$ & $2 / 19(10.5 \%)$ \\
\hline Estrogen therapy & $2 / 63(3.2 \%)$ & None \\
\hline No treatment & $2 / 86(2.3 \%)$ & $1 / 19(5.3 \%)$ \\
\hline IUD plus estrogen therapy & None & $3 / 25(12 \%)$ \\
\hline
\end{tabular}

To date, 194 of $223(87 \%)$ patients get pregnancy within $1 \mathrm{y}$ after surgery, in which $160(71.7 \%)$ patients have delivered healthy children. As shown in Table 3, successful pregnancy in groups A, B and C are 53, 45 and 62 cases, respectively. No significant difference is found between the 3 groups. Abortion in groups A, B and C are 10, 7 and 9 cases, respectively. Premature pregnancy in three groups is 2, 1 and 1 cases, with all infants have survived. Cesarean section cases in three groups are 26, 21 and 28 cases.

Statistical analysis shows that there are no statistical differences in postoperational adhesion, pregnancy rate, abortion rate, live birth rate and the rate of cesarean section ( $p>0.05$ ). However, the cost of group $\mathrm{C}$ is significantly lower than that in group A and group B.

\section{Discussion}

Nowadays, the hysteroscopic resection has been widely used in the treatment of uterine abnormalities, especially for uterine mediastinum. Compared with the traditional abdominal surgeries, hysteroscopic surgery possesses series of advantages including minor trauma, quick recovery, and less pregnancyrelated uterine rupture [13-15].

Commonly intrauterine adhesion is due to uterine trauma and infection. Intrauterine adhesion after TCRS is of particular concern, with the incidence of $2-5.3 \%$ in patients suffering TCRS. Several postoperational treatments have been applied as prophylactic approaches to prevent intrauterine adhesion, including placement of IUD and/or estrogen therapy.

However, the clinical efficacies of the post-TCRS IUD placement and estrogen therapy are controversial. Early in 1996, Dabirashrafi et al. questioned the value of estrogen therapy after hysteroscopic resection [16]. However, estrogen is still widely used to prevent uterine adhesion.

Theoretically, estrogen can accelerate the epithelialization of the exposed area after TCRS [17]. However, the probability of TCRS-related uterine cavity adhesion is very low due to the strong regeneration of the surrounding normal endometrial epithelium, where epithelial cells cover the exposed surgery 
area within 4-5 weeks after surgery. Thus, pregnancy can be expected in 6-8 weeks after surgery $[5,13]$.

Table 3. Patient follow-up.

\begin{tabular}{|c|c|c|c|c|c|c|c|c|c|}
\hline \multirow[t]{2}{*}{ Groups } & \multirow[t]{2}{*}{ Cases } & \multirow[t]{2}{*}{ Anti-adhesion } & \multirow[t]{2}{*}{ Cost (US\$) } & \multirow{2}{*}{$\begin{array}{l}\text { Adhesion } \\
(\%)\end{array}$} & \multirow[t]{2}{*}{ formation } & \multicolumn{4}{|c|}{ Pregnancy (\%) } \\
\hline & & & & & & Total & Live birth & C-section & Abortion \\
\hline A & 74 & IUD & 68 & $3(4.1 \%)$ & & $65(87.8 \%)$ & $53(81.5 \%)$ & $26(49.1 \%)$ & $10(15.4 \%)$ \\
\hline B & 63 & Climen & 30 & $2(3.2 \%)$ & & $55(87.3 \%)$ & $45(81.8 \%)$ & $21(46.7 \%)$ & $7(12.7 \%)$ \\
\hline C & 86 & None or Foley & $2.2^{* \#}$ & $2(2.3 \%)$ & & $74(86.0 \%)$ & $62(83.8 \%)$ & $27(43.5 \%)$ & $9(12.2 \%)$ \\
\hline
\end{tabular}

Niknejadi et al. showed that intrauterine adhesions occur in 5\% of patients with primary infertility, which is even higher than that in this study [4]. The postoperational intrauterine adhesion in this study reflects the normal reaction after surgery. The postoperational therapy used to prevent intrauterine adhesions does not decrease the occurrence of intrauterine adhesion formation. Proficient operation skills and avoiding unnecessary intrauterine operation may benefit patients rather than the postoperational therapy including IUD and/or ERT $[13,16]$.

In this study, the TCRS operation time is extended from 3-7 d to 3-10 d after menstruation. At this time, the endometrium gets slightly thinner and becomes moderately thick, but not too thick that may cause poor visibility. The endometrium at fundus uteri and opening of bilateral fallopian tubes are at the vigorous growth stage, which avoiding the occurrence of intrauterine adhesion. And then, the endometrium can cover the surgery area quickly after the operation. In addition, the patients have more time to make decision regarding the TCRS.

In this study, we utilize bipolar plasma kinetic system which causes relatively limited heating effect, less tissue damage and operation complications. Thus consequently, the postoperational scar becomes much smaller compared with the single-stage operation system. Postoperational hysteroscopic examination was usually conducted after the second menstruation following TCRS. Since no significant differences were observed in groups $\mathrm{A}, \mathrm{B}$ and $\mathrm{C}$, ultrasound examination can be performed on the first menstrual cycle or the third day of the second menstrual cycles and secretory phase to judge the effectiveness of the TCRS and hematomata rather than hysteroscopic examination. At present, we are conducting Transvaginal Ultrasonography (TVS) instead of HysteroSalpingography $(\mathrm{H} / \mathrm{S})$. If the postoperational TVS examination showed that mediastinal residues are greater than $1 \mathrm{~cm}$ or no pregnancy over one year after TCRS, H/S will be applied. The vast majority of patients received TCRS due to natural abortion or recurrent abortion. However, the recurrent spontaneous abortion may also be caused by polycystic ovary syndrome, hyperprolactinemia, inadequate luteal function, cervix relaxation, anti-cardiolipin antibody syndrome.

Even increasing amount of evidence indicates that vaginal delivery after TCRS is safe; most women with recurrent abortion are concerned about the risks the fatal may encounter during vaginal delivery. Thus they preferred to have cesarean section. This may explain the high cesarean section rates in Table 3, but the high cesarean section rate is due to "social factors", rather than "medical factors". Even with the placenta praevia combined with placenta implantation, complications are still comparable with normal pregnancy.

\section{Conclusions}

In short, neither postoperational IUD placement, nor estrogen therapy, demonstrated any discernible clinical benefits to the patients in preventing intrauterine adhesions and facilitating pregnancy after TCRS.

\section{Acknowledgment}

We thank Dr. Jifeng Bian (Colorado State University, USA) for the great help in the edit of the manuscript.

\section{Financial support}

The research was supported by a grant from the National Natural Science Foundation of China (No. 81270661).

\section{References}

1. Raga F, Bauset C, Remohi J, Bonilla-Musoles F, Simón C, Pellicer A. Reproductive impact of congenital Müllerian anomalies. Hum Reprod 1997; 12: 2277-2281.

2. Lin PC, Bhatnagar KP, Nettleton GS, Nakajima ST. Female genital anomalies affecting reproduction. Fertil Steril 2002; 78: 899-915.

3. Żyła MM, Wilczyński J, Nowakowska-Głąb A, Maniecka-Bryła I, Nowakowska D. Pregnancy and delivery in women with uterine malformations. Adv Clin Exp Med 2015; 24: 873-879.

4. Niknejadi M, Haghighi H, Ahmadi F. Diagnostic accuracy of transvaginal sonography in the detection of uterine abnormalities in infertile women. Iran J Radiol 2012; 9: 139-144.

5. Jayaprakasan K, Chan YY, Sur S, Deb S, Clewes JS, Raine-Fenning NJ. Prevalence of uterine anomalies and their impact on early pregnancy in women conceiving 
septum resection

after assisted reproduction treatment. Ultrasound Obstet Gynecol 2011; 37: 727-732.

6. Gubbini G, Di Spiezio Sardo A, Nascetti D. New outpatient sub-classification system for American fertility society classes V and VI uterine anomalies. J Minim Invasive Gynecol 2009; 16: 554-561.

7. Rackow BW, Arici A. Reproductive performance of women with müllerian anomalies. Curr Opin Obstet Gynecol 2007; 19: 229-237.

8. Bendifallah S, Faivre E, Legendre G, Deffieux X, Fernandez H. Metroplasty for AFS class V and VI septate uterus in patients with infertility or miscarriage: reproductive outcomes study. J Minim Invasive Gynecol 2013; 10: 1313-1321.

9. Tonguc EA, Var T, Yilmaz N, Batioglu S. Intrauterine device or estrogen treatment after hysteroscopic uterine septum resection. Int J Gynaecol Obstet 2010; 109: 226-229.

10. Dang Y, Qin Y, Tang R. Variants of the WNT7A gene in Chinese Patients with mullerian duct abnormalities. Fertil Steril 2012; 97: 391-394.

11. Xia M, Zhao H, Qin Y. LHX1 mutation screening in 96 patients with müllerian duct abnormalities. Fertil Steril 2012; 97: 682-685.

12. Tonguc EA, Var T, Yilmaz N, Batioglu S. Intrauterine device or estrogen treatment after hysteroscopic uterine septum resection. Int J Gynaecol Obstet 2010; 109: 226-229.
13. Valle RF, Ekpo GE. Hysteroscopic metroplasty for the septate uterus: review and meta-analysis. J Minim Invasive Gynecol 2013; 20: 22-42.

14. Munro MG, Christianson LA. Complications of hysteroscopic and uterine resectoscopic surgery. Clin Obstet Gynecol 2015; 58: 765-797.

15. Bhave Chittawar P, Franik S, Pouwer AW, Farquhar C. Minimally invasive surgical techniques versus open myomectomy for uterine fibroids. Cochrane Database Syst Rev 2014; 10: CD004638.

16. Dabirashrafi H, Mohammad K, Moghadami-Tabrizi N, Zandinejad K, Moghadami-Tabrizi M. Is estrogen necessary after hysteroscopic incision of the uterine septum? J Am Assoc Gynecol Laparosc 1996; 3: 623-625.

17. Scoccia B, Demir H, Elter K, Scommegna A. Successful medical management of post-hysteroscopic metroplasty bleeding with intravenous estrogen therapy: a report of two cases and review of the literature. J Minim Invasive Gynecol 2009; 16: 639-642.

\section{*Correspondence to}

Yulan $\mathrm{Mu}$

Department of Obstetrics and Gynecology

Shandong Provincial Hospital Affiliated to Shandong University

PR China 